\title{
Stability of behavioral estimates of activity-dependent modulation of pain
}

\author{
Meryl J Alappattu' \\ Mark D Bishop' \\ Joel E Bialosky' \\ Steven Z George ${ }^{1,2}$ \\ Michael E Robinson ${ }^{2,3}$ \\ 'Department of Physical Therapy, \\ University of Florida, Gainesville, \\ FL, USA; ${ }^{2}$ Center for Pain Research \\ and Behavioral Health, University \\ of Florida, Gainesville, FL, USA; \\ ${ }^{3}$ Department of Clinical and Health \\ Psychology, University of Florida, \\ Gainesville, FL, USA
}

This article was published in the following Dove Press journal:

Journal of Pain Research

24 May 20II

Number of times this article has been viewed

\begin{abstract}
Temporal sensory summation of pain (TSSP) is a proxy measure of windup in humans and results in increased ratings of pain caused by a repetitive, low-frequency noxious stimulus. Aftersensations (ASs) are pain sensations that remain after TSSP has been induced. We examined the within-session and across-session variability in TSSP and AS estimation in healthy participants and in participants with exercise-induced muscle pain in order to determine whether the presence of pain affected the stability of TSSP and ASs. TSSP was estimated by application of 10 repetitive, low-frequency $(<0.33 \mathrm{~Hz})$ thermal pulses and measured by the simple slope of pain ratings between the first and fifth pulses. ASs were measured by the presence of any remaining pain sensations up to 1 minute after TSSP was induced. TSSP estimation remained moderately stable in pain-free participants and in participants with pain within a single testing session but demonstrated low stability across sessions in pain-free participants. AS estimation was stable for all groups. Estimation of TSSP and ASs using these protocols appears to be a reliable single-session outcome measure in studies of interventions for acute muscle pain and in experimental studies with healthy participants. This article evaluates the reliability of a commonly used method of estimating TSSP and ASs in both healthy participants and in a clinically relevant model of acute pain. These protocols have the potential to be used as single-session outcome measures for interventional studies and in experimental studies.
\end{abstract}

Keywords: temporal sensory summation of pain, aftersensations, quantitative sensory testing, pain measurement reliability

Quantitative sensory testing (QST) is used to measure experimental pain sensitivity by applying chemical, mechanical, or thermal stimuli to muscles, viscera, or skin to determine thresholds or stimulus-response curves in humans. Recently, QST has been categorized as "static" and "dynamic". Static QST measures include threshold determination, representing the basal stage of the pain processing system and pain rating magnitude, which represents cerebral processing. In contrast, dynamic QST measures involve behavioral measures that represent central integration of the pain processing system, including temporal sensory summation of pain (TSSP) and descending inhibition of pain. ${ }^{1}$

Windup is a short-term, reversible central sensitization characterized by increased sensitivity of the dorsal horn neurons in response to repetitive, nociceptive stimuli. ${ }^{2}$ Price et $\mathrm{al}^{3}$ first proposed that TSSP in response to repetitive, thermal stimuli applied to the skin could be used as a proxy measure for central sensitization in humans. During windup, there is an increase in the excitability of nociceptive neurons in the dorsal horn of the spinal cord that occurs after exposure to persistent peripheral 
noxious stimuli or injury to the tissues or nerves. ${ }^{4}$ Previous studies have suggested that central sensitization may play a key role in the maintenance of chronic pain conditions such as irritable bowel syndrome, ${ }^{5}$ vulvar vestibulitis syndrome, ${ }^{6}$ and fibromyalgia..$^{7-9}$

TSSP is characterized by an increase in pain ratings at the end of the pulse train compared with those at the beginning and is thought to represent the excitatory capacity of the pain system. Aftersensations (ASs) refer to any pain sensations which remain after TSSP induction. ASs may reflect the intrinsic inhibition properties of the nociceptive system and have shown to be elevated in patients with fibromyalgia ${ }^{10}$ and nerve injury patients who experience allodynia. ${ }^{11}$ Prolonged pain after the removal of a painful stimulus may represent a reduced capacity to inhibit neuronal activity induced by TSSP. Since TSSP and ASs are potentially behavioral measures of central sensitization of pain enhanced in people with chronic pain, ${ }^{7,9-12}$ determining the effect of interventions on these measures could be useful in directing treatment for pain conditions. These measures are different from questionnaire-measures used to assess outcomes because they allow investigators to better understand the effects of interventions related to specific pain processing mechanisms of the body.

Different protocols for inducing, measuring, and calculating TSSP exist. The reliability of session-to-session repetitive-phasic (20 pulse) and tonic methods of inducing TSSP in healthy volunteers has been tested. ${ }^{13}$ In that study, TSSP was calculated by the absolute and percentage changes between first and last pain ratings, in addition to logarithmic regression curves based on consecutive ratings given by each subject. Other authors have induced TSSP in healthy participants and in participants with pain using fewer thermal stimuli; 10 or 6 pulses, for example.,12,14-16

We planned to extend work from Granot et al ${ }^{13}$ by examining the stability of TSSP and AS estimates. We had two primary aims. First, we sought to evaluate the stability of TSSP and AS responses within and across sessions in healthy participants. Using healthy participants allowed us to examine pain modulation pathways without the interference of a clinical pain condition. Second, we sought to evaluate the stability of TSSP and ASs in individuals experiencing acute, exercise-induced musculoskeletal pain in order to draw inferences about how musculoskeletal pain may affect aspects of pain sensitivity in a controlled environment. We hypothesized 1) within- and across-session TSSP and AS estimates would be at least moderately stable in pain-free participants and in those with exercise-induced musculoskeletal pain, and
2) the presence of musculoskeletal pain would negatively influence the stability of TSSP and ASs.

\section{Materials and methods Participants}

A convenience sample was pooled from previously reported studies that focused on thermal pain responses ${ }^{15,17}$ and a recently completed exercise-induced pain study that has not been previously reported. All data were collected in the same laboratory using the same equipment and QST protocols. The current manuscript represents the findings of a planned, secondary analysis of these studies. Each study was approved by the Institutional Review Board at the University of Florida, and all participants provided informed consent to participate in the studies.

\section{Quantitative sensory testing protocol}

Thermal stimuli were delivered with the Medoc Neurosensory Analyzer (TSA- 2001, Ramat Yishai, Israel). Participants underwent a practice session in order to familiarize themselves with the thermal stimuli and the rating system. First, threshold stimuli were rated with a numerical pain rating scale (NPRS) ranging from 0 (no pain) to 100 (worst pain imaginable). Next, participants were introduced to the TSSP, where a train of 4 consecutive thermal pulses was applied to the plantar surface of the nondominant foot at an interstimulus interval of $<0.33 \mathrm{~Hz}$. The temperature rose rapidly from a baseline of $40^{\circ} \mathrm{C}$ to a peak of $50^{\circ} \mathrm{C}$. The participants were instructed as follows:

\footnotetext{
"We are interested in you letting us know if you feel the "second pain" associated with the range of pain. Second pain is best described as the delayed pain intensity felt after the initial onset of heat. For example, it is the sensation you feel after you have removed your hand from a hot surface, but still experience a delayed surge of pain. We will be asking you to rate your second pain in specific parts of this study and want you to practice that now using the numerical rating system with 0 being no pain and 100 being the most intense pain sensation imaginable".
}

Following the practice session, TSSP was assessed through the application of a train of 10 consecutive thermal pulses applied to the plantar surface of the nondominant foot at an interstimulus interval of $<0.33 \mathrm{~Hz}$. The temperature rose rapidly $\left(10^{\circ} \cdot \mathrm{s}^{-1}\right)$ from a baseline of $40^{\circ} \mathrm{C}$ to a peak of $50^{\circ} \mathrm{C}$. The participants were asked to rate the magnitude of their delayed (second) pain sensation using the NPRS following each pulse. ASs were measured by asking participants to rate 
any pain sensation using the NPRS that remained 15, 30, 45, and 60 seconds after cessation of the 10th pulse (Figure 1).

\section{Study \#I -Within-session stability}

For the first study, within-session testing occurred during a single testing session with the previously described thermal QST protocol repeated at an interval ranging between 2 and 10 minutes. Data from 210 pain-free participants were included in Study \#1.

\section{Study \#2 - Across-session stability}

For the second study, data used to test across-session variability were collected 2 weeks apart with the previously described thermal QST protocol. Data from 35 pain-free participants were included in Study \#2.

\section{Study \#3 - Induction of musculoskeletal pain and its effects on stability}

The induction of musculoskeletal pain was performed on 52 participants in the third study. Prior to exercise, all participants completed a warm-up session consisting of riding the stationary bicycle at a speed of 50-60 rpm and $1 \mathrm{Kp}$ of resistance and static passive stretching of the lower extremities. Each participant performed an isometric test of total torque through their available trunk flexion range of motion (ROM) using a MedX lumbar extension exercise machine following the standardized protocol. ${ }^{18}$ After baseline torque was recorded, participants performed bouts of dynamic exercise to the point of volitional fatigue. To perform the dynamic fatiguing exercise bout, the participants were seated and restrained in a MedX lumbar extension exercise machine. Participants performed as many repetitions as possible using a weight load equal to approximately $80 \%$ of the peak torque measured during the isometric test. Each repetition

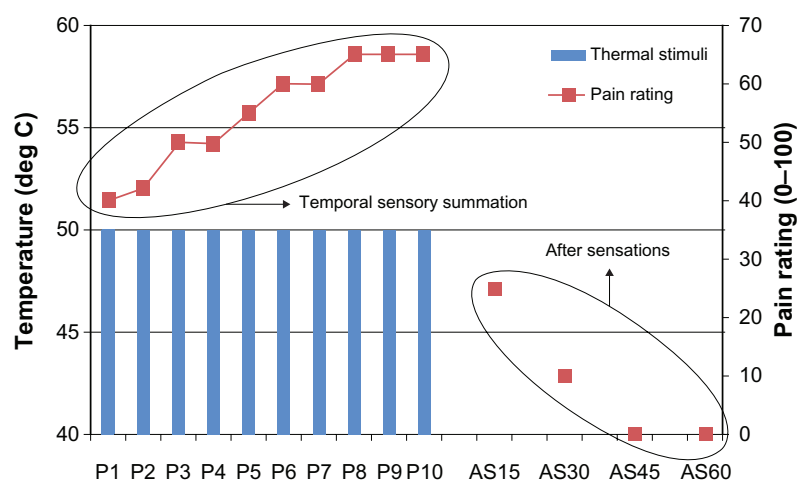

Figure I Theoretical model of TSSP and AS.

Abbreviations: AS, aftersensation; $\mathrm{P}$, pain; TSSP, temporal sensory summation of pain. was performed through the full available ROM. Repetitions of both exercises continued until the patient reported being unable to move through a full ROM (volitional fatigue). At the end of the exercise set, the isometric torque test was performed again. Participants repeated the sequence of dynamic exercise and static testing until total measured torque decreased to $50 \%$ of the baseline measurement. Participants were instructed not to initiate any medication, or apply any intervention to the lumbar spine. Forty-eight hours after the exercise protocol, participants returned to the laboratory and underwent the TSSP and AS protocols in a single session as described above.

\section{Data analysis}

TSSP magnitude was calculated using two methods: 1) a change score was calculated as the difference between the ratings of the first and fifth pulse, and 2) simple regression coefficients were calculated based on the ratings of pulses $1-10$ and on ratings $1-5$. The first method was chosen because it is a simple arithmetic calculation which is likely to be performed easily in practice. Given the anecdotal observations of fluctuation from pulse to pulse, we used simple regression coefficients to determine the variance of the slope across the 10 pulses.

For this study we used the rating of any AS at 15 seconds post-TSSP protocol as the variable of interest because none of the participants reported ASs at the 30-, 45-, or 60-second mark, which is consistent with other data that have examined after sensations in healthy volunteers. ${ }^{10-11}$

We examined all measures (TSSP and ASs) for systematic bias from test to test using $t$-tests. ${ }^{19}$ Normality was visually explored with Normal Q-Q plots and tested with the Kolmogorov-Smirnov normality test. The magnitude of the test to test variation in rating was assessed by calculating the standard error of measurement (SEM), using the square root of the mean square error term from a random-effects model ANOVA. ${ }^{20}$ The use of the SEM, because it assumes a normal distribution of error, requires that the measurement error is not related to the magnitude of the measured variable. Heteroscedastic data shows that individuals who score the highest in a particular test also show the greatest amount of measurement error. Heteroscedasticity was formally examined by plotting the absolute differences between testing sessions, against the mean score of both testing sessions. Additionally, Spearman's rho correlation was used to rule out a relationship between each individual's absolute score difference and his or her mean. ${ }^{21}$ Last, we calculated two-way random effects intraclass coefficients (ICCs) for each of the measures. 
All data were analyzed using SPSS for Windows Version 17 or SAS 9.2.

\section{Results}

\section{Study \#I - Within-session: pain free participants}

The average age of participants in the pooled dataset for this set of analyses was $23.7 \pm 3.9$ years, and $136(64.7 \%)$ of them were women. No differences between test sessions for any measure of TSSP or for ASs $(P=0.26)$ was noted. Scatterplots of the data from each test session did not suggest outliers or influential points within the data (Figure 2). However, there was evidence of heteroscedasticity in the error terms associated with the simple change score, with larger errors associated with greater values of the TSSP, and the distribution of the errors deviated from a theoretical normal distribution $(P=0.03)$ (Table 1$)$.

The within-session ICCs for TSSP ranged from 0.41 to 0.67 with an SEM ranging from 1.7 to 13.0. For ASs, the within-session ICC was 0.82 with an SEM of 6.5 (Table 2).

\section{Study \#2 - Across-session: pain free participants}

The average age of these participants was $24.3 \pm 3.1$ and there were $26(74.2 \%)$ women. No evidence of bias or heteroscedasticity was noted across sessions (Table 1). Across-session
ICC values were generally lower than within-session values for all measures (Table 2), indicating lower reliability acrosssession.

\section{Study \#3 - Within-session: participants with exercise-induced acute pain}

The average age of these participants was $22.4 \pm 5.7$ years, and there were 24 (46.1\%) women. Forty-eight hours after pain induction ratings of spontaneous pain at rest ranged from 10 to 68 , using a $100 \mathrm{~mm}$ visual analog scale. No differences between test sessions for any measure of TSSP or for ASs $(P>0.34)$ was noted. Tests for heteroscedasticity were negative. The ICC for TSSP ranged from 0.29 to 0.76 , demonstrating a wide range of reliability. The regression coefficient of ratings $1-5$ was not statistically reliable (confidence interval included zero). The ICC for ASs remained above 0.8 (Table 2), indicating that it was stable within-session.

\section{Discussion}

Our results indicate that ratings of pain induced with our TSSP protocol were moderately stable ${ }^{22}$ within a single testing session for both pain-free (Study \#1) and induced musculoskeletal pain (Study \#3) participants but demonstrated low stability across a 2-week time period in healthy participants (Study \#2). AS measurements were highly stable within- and across-session for all three studies.
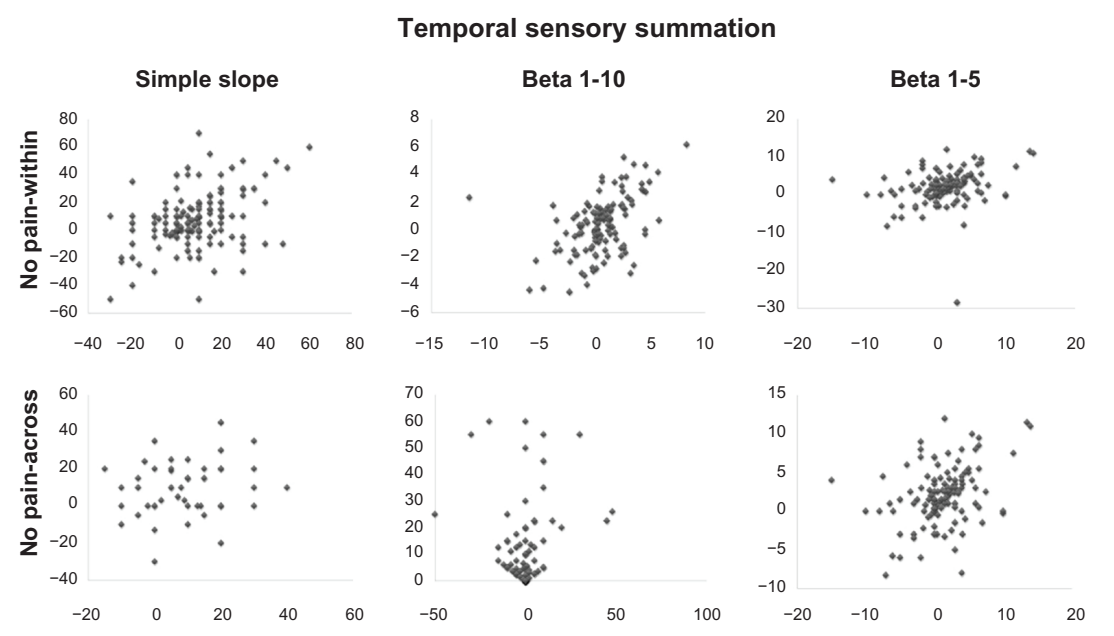

\section{After sensations}
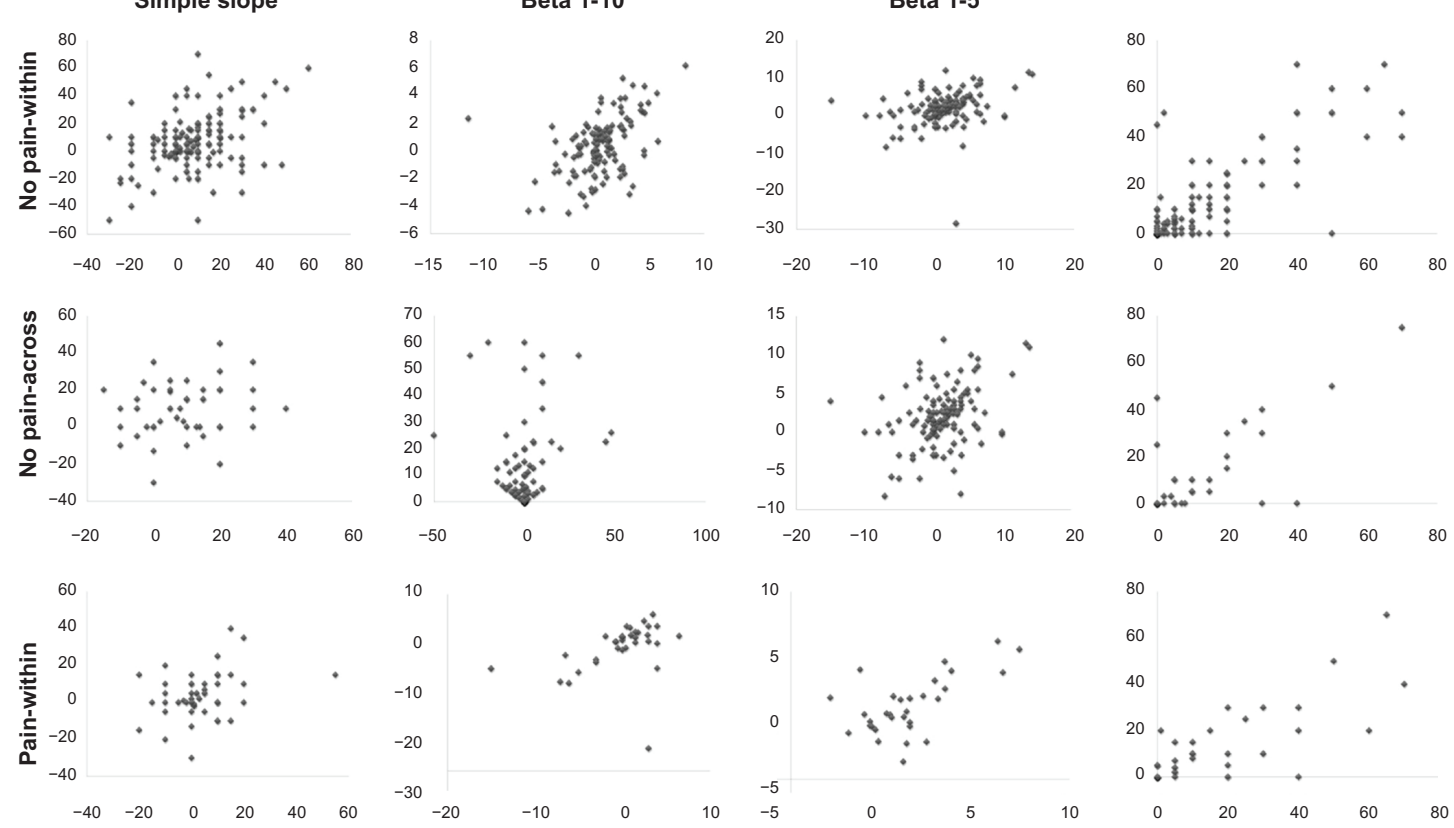

Figure 2 These scatter plots represent testing session I (x-axis for all) compared to testing session 2 ( $y$-axis for all). The units of the simple slope graphs (temporal summation) represent change in a $10 \mathrm{I}$-point numeric rating scale scores calculated by subtracting the rating at pulse 5 from the rating at pulse I. The 'Beta' graphs are of regression coefficients and are unitless. After sensations are rated using the I0I-point numeric rating scale. 
Table I Correlations of change score to mean of the trials (heteroscedasticity)

\begin{tabular}{llllll}
\hline & & Within & & Across \\
\cline { 3 - 4 } & & Pain free & Acute pain & & Pain free \\
\hline TSSP & Change & 0.188 & 0.078 & 0.101 \\
& Beta I-10 & 0.204 & 0.033 & \\
& Beta I-5 & 0.015 & 0.066 & \\
ASs & & -0.020 & -0.214 & & 0.010 \\
\hline
\end{tabular}

Abbreviations: AS, after sensation; TSSP, temporal sensory summation of pain.

These results suggest that TSSP and AS measurements using these protocols could be potentially useful in assessing changes in pain sensitivity within a single testing session for both experimental studies involving healthy participants and, potentially, in clinical research with participants who have musculoskeletal pain. Our data support the hypothesis that the presence of musculoskeletal pain did not affect the stability of TSSP and ASs.

Given the individual variability in pain sensation and experience, methods of assessing change in pain sensitivity must be stable from measurement to measurement in order to validate that the observed change was directly related to pain sensitivity and not due to a fluctuation in the measurement tool. Another important aspect of measurement reliability is the degree of observed change; how much change in pain sensitivity must occur in order to ensure that change, not measurement error, led to that change? The SEM observed in our TSSP calculation was 10 and above for all groups, which may have been due to the lack of participants' familiarity with the protocol. At the $95 \%$ confidence level, the "minimal detectable change" in pain rating is approximately 19.
Minimal detectable change represents the amount of change that would need to occur in order to be confident that actual change in TSSP occurred as opposed to measurement error. Practice sessions of the full protocol prior to actual TSSP induction and measurement could potentially reduce the magnitude of this error. Future studies should investigate whether these extra sessions reduce this error.

To the best of our knowledge, one other study ${ }^{13}$ has been published to date that examines the stability of TSSP using thermal stimuli in healthy participants. Similar to our current study, the investigators in this study examined the acrosssession reliability of temporal summation measurement using thermal stimuli. They tested both repetitive-phasic (20 pulses, inter-stimulus interval of 2.5 seconds) and tonic stimuli (60 seconds). They determined the stimulus intensity by determining the temperature at which each subject rated their pain level at 60/100 using the NPRS. This temperature, "pain-60", was used to induce TSSP, and the authors found that using it led to a smaller range of pain scores, reduced floor and ceiling effects, and induced TSSP in most participants. They also found that no significant differences existed in across-session (1 week) TSSP induction using phasic and tonic stimuli protocols, but concluded the protocols may be more applicable if used in group testing instead of using them for individual subject data, given the variability of data across time.

In an attempt to determine the influence of musculoskeletal pain, we also tested these measures in a clinically relevant model of experimental pain and found that they remained moderately stable within a single testing session. This approach was selected to investigate the potential

Table 2 Summary of results of ICC and SEM calculations

\begin{tabular}{|c|c|c|c|c|c|}
\hline & \multicolumn{3}{|l|}{ TSSP } & & \multirow[t]{2}{*}{ AS } \\
\hline & Change score & Beta $1-10$ & Beta I-5 & & \\
\hline \multicolumn{6}{|l|}{ Within } \\
\hline \multirow[t]{4}{*}{ Pain free $(N=210)$} & Mean & 7.8 & 0.5 & 1.5 & 9.5 \\
\hline & ICC & 0.58 & 0.62 & 0.41 & 0.82 \\
\hline & $95 \% \mathrm{Cl}$ & $0.4 I, 0.67$ & $0.46,0.73$ & $0.16,0.59$ & $0.76,0.86$ \\
\hline & SEM & 13 & 1.7 & 3.7 & 6.5 \\
\hline \multirow[t]{4}{*}{ Acute pain $(\mathrm{N}=52)$} & Mean & 3.8 & 1.7 & 2.2 & 11.8 \\
\hline & ICC & 0.49 & 0.76 & 0.29 & 0.86 \\
\hline & $95 \% \mathrm{Cl}$ & $0.29,0.76$ & $0.5 \mathrm{I}, 0.88$ & $0.0,0.63$ & $0.75,0.92$ \\
\hline & SEM & 10 & I.I & 3.4 & 8 \\
\hline \multicolumn{6}{|l|}{ Across } \\
\hline \multirow[t]{4}{*}{ Pain free $(\mathrm{N}=35)$} & Mean & 9.2 & 0.3 & 2.2 & 11.8 \\
\hline & ICC & 0.37 & 0.54 & 0.3 & 0.75 \\
\hline & $95 \% \mathrm{Cl}$ & $0.0,0.66$ & $0.09,0.77$ & $-0.34,0.63$ & $0.59,0.85$ \\
\hline & SEM & 13 & 3.6 & 3.5 & 8.3 \\
\hline
\end{tabular}

Abbreviations: AS, after sensation; $\mathrm{Cl}$, confidence interval; ICC, intraclass coefficient; SEM, standard error of measurement; TSSP, temporal sensory summation of pain. 
clinical translation of pain sensitivity testing protocols to estimate pain sensitivity in patients with acute musculoskeletal pain. The protocols use simple change scores and are time-efficient, which makes them ideal for measuring change in a clinical setting. The ability to measure change in pain sensitivity may provide a better understanding of how particular interventions may work to alter pain processing mechanisms. Currently, many clinical pain outcomes are assessed with the use of standardized, validated measures of sensory and affective dimensions of pain, such as the visual analog scale, NPRS, and McGill Pain Questionnaire. ${ }^{23}$ While these tools are useful in measuring the sensory and affective aspects of pain, they lack the ability to directly quantify pain sensitivity that may be mediated by the central nervous system, including ascending and descending modulation and/ or inhibition of pain. The use of thermal stimuli to reliably quantify pain sensitivity may allow practitioners to observe the excitatory (TSSP) and inhibitory (AS) capabilities of the pain system in people with acute pain within a single testing session. However, further work remains to determine what degree of change in pain sensitivity may be clinically meaningful.

In experimental settings, the TSSP and AS protocols examined in this study may allow future investigators to reliably examine immediate changes in pain sensitivity in response to a treatment in intervention studies. Given the high reliability of ASs between testing sessions, it may be used to detect changes in inhibitory pain mechanisms across time. These protocols may also be more appropriate to measure differences in pain sensitivity in pain-free participants compared with those with pain in order to better understand pain processing mechanisms.

\section{Limitations}

A limitation to calculating TSSP using a simple change score appears to be in the variability of the estimate of TSSP for participants with large values (greater summation). Potentially, those individuals who present with larger magnitudes of TSSP will require greater change in TSSP to represent true change in TSSP. This heteroscedasticity is eliminated when using regression coefficients to estimate the magnitude of the slope of the ratings indicating more consistent variation across the range of values of TSSP.

This study included data from pain-free participants and participants with exercise-induced musculoskeletal pain. The generalizability of these results may be appropriate for people with acute muscle pain, but not for those with chronic muscle pain. Thermal stimuli were used to induce
TSSP and ASs, so we are unable to generalize these results with the use of mechanical or chemical stimuli. Finally, the temporal stability of static measures such as threshold or pain-rating magnitude in the groups were not addressed and therefore we are unable to conclude how the stability of the static QST measures compare to the dynamic QST measures used in this study.

\section{Conclusion}

Our data support the use of this TSSP protocol to reliably measure responses within a single testing session, in addition to the use of AS measurement within- and across-session. Based on our results, measuring pain inhibition with ASs appears to be more stable immediately and over time compared with measuring pain facilitation with TSSP. Further work remains to establish how much change needs to be observed in order to be distinguished from measurement error and to be considered clinically meaningful.

\section{Acknowledgments}

This work was supported in part by funding from: National Institutes of Health K01AR054331-01, R21AT002796-01, 5K12HD055929-02, T32HD043730, The Foundation for Physical Therapy, and the University of Florida Open-Access Publishing Fund.

\section{Disclosure}

The authors report no conflicts of interest in this work.

\section{References}

1. Arendt-Neilsen L, Yarnitsky D. Experimental and clinical applications of quantitative sensory testing applied to skin, muscles, and viscera. J Pain. 2009;10:556-572.

2. Woolf CJ. Windup and central sensitization are not equivalent. Pain. 1996;66:105-108.

3. Price DD, HU JW, Dubner R, Gracely RH. Peripheral suppression of first pain and central summation of second pain evoked by noxious heat pulses. Pain.1977;3:57-58

4. Ji RR, Kohno T, Moore KA, Woolf CJ. Central sensitization and LTP: do pain and memory share similar mechanisms? Trends Neurosci. 2003;26:696-705.

5. Price DD, Zhou Q, Moshiree B, Robinson ME, Verne GN. Peripheral and central contributions to hyperalgesia in irritable bowel syndrome. $J$ Pain. 2006; 7:529-535.

6. Granot M, Friedman M, Yarnitsky D, Zimmer EZ. Enhancement of the perception of systemic pain in women with vulvar vestibulitis. BJOG. 2002;109:863-866.

7. Price DD, Staud R, Robinson ME, Mauderli AP, Cannon R, Vierck CJ. Enhanced temporal summation of second pain and its central modulation in fibromyalgia patients. Pain. 2002;99:49-59.

8. Staud R, Domingo M. Evidence for abnormal pain processing in fibromyalgia syndrome. Pain Med. 2001;91:208-215.

9. Staud R, Vierck CJ, Cannon RL, Mauderli AP, Price DD. Abnormal sensitization and temporal summation of second pain (wind-up) in patients with fibromyalgia syndrome. Pain. 2001;91:165-176. 
10. Staud R, Robinson ME, Price DD. Temporal summation of second pain and its maintenance are useful for characterizing widespread central sensitization of fibromyalgia patients. J Pain. 2007;8:893-901.

11. Gottrup H, Kristensen AD, Bach FW, Jensen TS. Aftersensations in experimental and clinical hypersensitivity. Pain. 2003;103:57-64

12. Staud R, Bovee CE, Robinson ME, Price DD. Cutaneous c-fiber abnormalities of fibromyalgia patients are specifically related to temporal summation. Pain. 2009;139:315-323.

13. Granot M, Granovsky Y, Sprecher E, Nir RR, Yarnitsky D. Contact heatevoked temporal summation: tonic versus repetitive-phasic stimulation. Pain. 2006;122:295-305.

14. Beneciuk JM, Bishop MD, George SZ. Effects of upper extremity neural mobilization on thermal pain sensitivity: a sham-controlled study in asymptomatic participants. J Orthop Sports Phys Ther. 2009;39:428-438.

15. George SZ, Bishop MD, Bialosky JE, Zeppieri G, Robinson ME. Immediate effects of spinal manipulation on thermal pain sensitivity: an experimental study. BMC Musculoskelet Disord. 2006 Aug 15; 7:68.

16. Staud R, Price DD, Fillingim RB. Advanced continous-contact heat pulse design for efficient temporal summation of second pain (windup). J Pain. 2006;7:575-582.
17. Bialosky JE, Bishop MD, Robinson ME, Barabas JA, George SZ. The influence of expectation on spinal manipulation induced hypoalgesia: an experimental study in normal subjects. BMC Musculoskelet Disord. 2008 Feb 11;9:19.

18. Graves JE, Pollock ML, Carpenter DM, et al. Quantitative assessment of full range-of-motion isometric lumbar extension strength. Spine. 1990;15:289-294.

19. Bland JM, Altman DG. Measuring agreement in method comparison studies. Stat Methods Med Res. 1999;8:135-160.

20. Stratford PW, Goldsmith CH. Use of the standard error as a reliability index of interest: an applied example using elbow flexor strength data. Phys Ther. 2007;77:745-750.

21. Atkinson G, Nevill AM. Statistical methods for assessing measurement error (reliability) in variables relevant to sports medicine. Sports Med. 1998;26:217-238.

22. Rosner B. Fundamentals of Biostatistics. 6th ed. Belmont: Thompson Learning; 2006:613-617.

23. Breivik H, Borchgrevink PC, Allen SM, et al. Assessment of pain. $B r$ J Anaesth. 2008;101:17-24.
Journal of Pain Research

\section{Publish your work in this journal}

The Journal of Pain Research is an international, peer-reviewed, open access, online journal that welcomes laboratory and clinical findings in the fields of pain research and the prevention and management of pain. Original research, reviews, symposium reports, hypothesis formation and commentaries are all considered for publication.

\section{Dovepress}

The manuscript management system is completely online and includes a very quick and fair peer-review system, which is all easy to use. Visit http://www.dovepress.com/testimonials.php to read real quotes from published authors. 